\title{
Cytologic Diagnosis of Biliary Strictures: FISH or Cut the Sensitivity Rate?
}

\author{
Douglas G. Adler ${ }^{1}$ - Benjamin Witt ${ }^{2}$
}

Published online: 12 January 2018

(c) Springer Science+Business Media, LLC, part of Springer Nature 2018

The definitive diagnosis of a malignant biliary stricture remains difficult, even if cancer is strongly suspected. Whereas some subsets of patients are relatively easy to diagnose (i.e., those with pancreatic masses causing extrahepatic biliary obstruction), many patients can defy even the most exhaustive diagnostic efforts. Specifically, cholangiocarcinoma arising in patients with and without primary sclerosing cholangitis (PSC) can be extremely difficult to diagnose, since these lesions are often hypocellular due to fibrosis and an underlying desmoplastic reaction. These tumor features can humble even the most aggressive, perceptive, and experienced biliary endoscopist and pathologist.

In patients with a malignant biliary stricture, it is often helpful to employ multiple technologies in an attempt to fully evaluate and sample relevant tissues. Although endoscopic retrograde cholangiopancreatography (ERCP) with routine biliary brushings is widely used, advanced adjunctive techniques such as cholangioscopy with intraductal biopsies and/or endoscopic ultrasound (EUS) with fine needle aspiration (FNA) or fine needle biopsy (FNB) of suspicious periductal nodes or other concerning lesions are less often utilized.

Fluorescence in situ hybridization (FISH) is an adjunctive test that can be performed on biliary brushing cytologic samples in an effort to confirm the presence of malignant cells. FISH, originally developed in the 1980s as a means of detecting complementary DNA sequences in intact chromosomes [1], has been adapted in the past decade to clinical diagnosis, mostly in tertiary referral centers [2]. FISH utilizes fluorescently labeled probes that hybridize

Douglas G. Adler

Douglas.adler@hsc.utah.edu

1 Gastroenterology and Hepatology, University of Utah School of Medicine, Huntsman Cancer Center, 30N 1900E 4R118, Salt Lake City, UT 84132, USA

2 Department of Pathology, University of Utah School of Medicine, Salt Lake City, UT, USA with the nuclear DNA of individual cells in a cytologic sample obtained from brushings of a biliary stricture. The most commonly clinically used FISH-based test adopts the UroVysion $^{\mathrm{TM}}$ test, originally developed for detecting urothelial carcinoma in voided urine specimens, to biliary brushings. The FISH probes detect polysomy of chromosomes 3, 7, and 17 and may also include detection of deletion of chromosome locus 9p21, genetic abnormalities typical of many types of epithelial cancers, including those affecting the biliary epithelium. FISH testing can be performed on any standard cytologic specimen obtained by ERCP, with testing possible locally or by reference laboratories. FISH should be interpreted by an experienced cytopathologist, since the test requires paired morphologic assessment of the specimen in order to ensure that pertinent chromosomal abnormalities occur within morphologically abnormal biliary epithelial cells (rather than in overlapping normal cells or in inflammatory cells). At our center, we routinely perform FISH in order to increase the sensitivity and specificity of biliary cytology.

Multiple reports have demonstrated the value of adding FISH to routine brush cytology in patients with biliary strictures in patients with and without PSC [2-7]. The primary gain with supplementary FISH is an increase in the sensitivity of detecting cholangiocarcinoma without reducing specificity. Another advantage is that FISH can better determine lesions that are deemed "suspicious for malignancy" in that a positive FISH result would support cholangiocarcinoma, whereas a negative FISH is more consistent with a benign/reactive lesion. Furthermore, prior stenting is a widely recognized limitation for biliary brush cytology as the degree of reactive atypia in this setting can closely mimic carcinoma. The cytomorphologic overlap can be so deceptive that some cytopathologists will not render a malignant diagnosis on a recently stented patient [8]. Many feel that FISH co-testing in this setting contributes to optimal patient care as it increases sensitivity to a reasonable range (in the current study, the sensitivity of brushing cytology 
was only $20 \%$ in the setting of recently stented patients) to better inform patient management. Lastly, with the recent finding that chromosomal abnormalities paralleling those in cholangiocarcinoma can be detected in premalignant lesions such as biliary dysplasia, FISH should not be used as a standalone test. Thus, co-testing, or pairing FISH with cytology, is optimal so as not to create false-positive FISH results. By extension, a positive FISH result with a negative concurrent cytology interpretation should be treated with caution [9].

In this issue of the Digestive Diseases and Sciences, Brooks et al. [10] reported the results of a large, retrospective study of patients with biliary strictures who underwent FISH compared with EUS-assisted FNA and cholangioscopic biopsies. The final analysis included 281 patients, 42 of whom had PSC. The authors reported that routine brush cytology had a sensitivity of $35 \%$ and a specificity of $99 \%$ when it included the presence of suspicious or malignant cells. When they included only malignant cells the sensitivity fell to a distressing 26\% although specificity was largely unchanged at $100 \%$. FISH, when focused on polysomy of chromosomes 3,7 , and 17, had a sensitivity and specificity of 45 and $99 \%$, respectively. When the authors also included loci 9p21 heterozygous or homozygous deletions as a positive result, FISH sensitivity increased to 55\% while maintaining a specificity of $99 \%(p=0.001)$. Furthermore, when brushings showed malignant or suspicious cells and FISH revealed a 9p21 deletion, sensitivity rose to $66 \%$ in those with a prior biliary stent and to $62 \%$ in those without a prior stent. EUS FNA and cholangioscopic biopsies were both deemed important supplementary tests when performed in concert with routine brush cytology and FISH, although cholangioscopic biopsies were borderline significantly beneficial when compared to FISH.

The principal message of the study by Brooks et al is that FISH can add significantly to the diagnostic investigation of patients with biliary strictures using standard biliary brush catheters. The few additional minutes needed to take such brushing can significantly augment diagnostic accuracy. If FISH testing is not available onsite, the sample can be sent out to any one of several large national pathology laboratories. Sadly, although the technology is not new, currently few endoscopists use FISH in their routine clinical practice. It is hoped that the study by Brooks et al will renew interest in the adoption of FISH testing to the routine diagnosis of biliary strictures and help make this test a staple, and not a rarity, of biliary endoscopy.

\section{References}

1. Langer-Safer PR, Levine M, Ward DC. Immunological method for mapping genes on Drosophila polytene chromosomes. Proceedings of the National Academy of Sciences. 1982;79:4381-4385.

2. Kipp BR, Stadheim LM, Halling SA, et al. A comparison of routine cytology and fluorescence in situ hybridization for the detection of malignant bile duct strictures. Am J Gastroenterol. 2004;99:1675-1681.

3. Smoczynski M, Jablonska A, Matyskiel A, et al. Routine brush cytology and fluorescence in situ hybridization for assessment of pancreatobiliary strictures. Gastrointest Endosc. 2012;75:65-73. https://doi.org/10.1016/j.gie.2011.08.040.

4. Barr Fritcher EG, Voss JS, Jenkins SM, et al. Primary sclerosing cholangitis with equivocal cytology: fluorescence in situ hybridization and serum CA 19-9 predict risk of malignancy. Cancer Cytopathol. 2013;121:708-717. https://doi.org/10.1002/cncy.2133 1.

5. Boldorini R, Paganotti A, Andorno S, et al. A multistep cytological approach for patients with jaundice and biliary strictures of indeterminate origin. J Clin Pathol. 2015;68:283-287. https://doi. org/10.1136/jclinpath-2014-202731.

6. Liu R, Cox K, Guthery SL, et al. Cholangiocarcinoma and highgrade dysplasia in young patients with primary sclerosing cholangitis. Dig Dis Sci. 2014;59:2320-2324. https://doi.org/10.1007 /s10620-014-3152-0.

7. Huddleston BJ, Lamb RD, Gopez EV, Adler DG, Collins BT. Cholangiocarcinoma in a 17-year-old boy with primary sclerosing cholangitis and UroVysion ${ }^{\mathrm{TM}}$ fluorescent in situ hybridization. Diagn Cytopathol. 2012;40:337-341. https://doi.org/10.1002 /dc. 21629.

8. Cibas ES, Ducatman BS. Cytology: Diagnostic Principles and Clinical Correlates. 4th ed. Amsterdam: Elsevier; 2014:404.

9. Kerr SE, Barr Fritcher EG, Campion MB, et al. Biliary dysplasia in primary sclerosing cholangitis harbors cytogenetic abnormalities similar to cholangiocarcinoma. Human Pathology. 2014;45:1797-1804.

10. Brooks, C, Gausman, V, Kokoy-Mondragon, C, et al. The role of fluorescent in-situ hybridization, cholangioscopic biopsies and EUS/FNA in the evaluation of biliary strictures. Dig Dis Sci. (Epub ahead of print). https://doi.org/10.1007/s10620-017-4824 -3 . 